\title{
Long-Wave Infrared Functional Brain Imaging in Human: A Pilot Study
}

\author{
Christian C Joyal ${ }^{1,2, *}$ and Mylene Henry ${ }^{2}$ \\ ${ }^{1}$ Université du Québec à Trois-Rivières, Canada \\ ${ }^{2}$ Institut Philippe-Pinel de Montréal, Canada
}

\begin{abstract}
Although some authors suggest to use Long-Wave Infrared (LWIR) sensors to evaluate brain functioning, the link between emissions of LWIR and mental effort is not established. The goal of this pilot study was to determine whether frontal LWIR emissions vary during execution of neuropsychological tasks known to differentially activate the pre-frontal cortex (simple color presentations, induction of the Stroop effect, and a gambling task with real money). Surprisingly, LWIR emissions as measured with bilateral frontal sensors in 47 participants significantly differed between tasks, in the supposed direction (Color $<$ Stroop $<$ Gambling), in spite of counterbalanced presentations. This pilot study suggests that investigations of convergent validity with other types of brain imaging techniques can be initiated with LWIR imaging. If confirmed, this technique would offer a simple and accessible method to evaluate frontal cortex activation.
\end{abstract}

Keywords: Long-wave, passive, infrared, brain imaging.

\section{INTRODUCTION}

Infrared (IR) light is electromagnetic radiation of wavelengths invisible to the human eye $(0.75$ to $300 \mu \mathrm{m})$. Long-Wave Infrared (LWIR) emissions represent a IR specific spectrum (8 to15 $\mu \mathrm{m})$ associated with thermal modification. The development of infrared cameras and sensors sensitive to LWIR allows Infrared Thermography (IRT), a temperature-based imaging technique used in different domains, including military (e.g. target acquisition, guiding missile technology, night human detection), industrial (e.g. thermal efficiency analyses, remote temperature sensing, localization of over-heating parts), domestic (e.g. alarm systems and movement detection), and emergency services (e.g. nonvisual and distant localization of fire bases, heat leaks, warm-blooded animal; see [1] for a review). IRT is also used in medicine as a diagnostic aid for vascular, tumor, or cancer-related pathologies [2, 3]. Given that brain activation is associated with small variations of local cerebral blood flow [4] and thermal radiation [5], it is theoretically possible to use IRT as a crude technique of brain functional imaging [6]. This idea is certainly not new [7], but it was abandoned because of methodological difficulties [8]. More recently, some authors proposed the use of infrared cameras to deduce human brain functioning from temperature variation of the scalp $[6,9,10]$, and multiple-contact [11] or single non-contact [12] infrared head-mounted sensors have also been used. Still, the link between functional activation of the cortex and temperature modification of the scalp is not established. Temperature increases of the skin might be associated with other factors, including ambient temperature, peripheral activation, waking

*Address correspondence to this author at the Université du Québec à TroisRivières, Canada; Tel: 819-376-5011; Fax: 819-376-5195;

E-mail: christian.joyal@uqtr.ca state, etc. Another issue is that functional cortical $\mathrm{T}$ increases might be less localised than previously believed, affecting adjacent areas as well [13]. Thus, before conducting concomitant or construct validity studies with the LWIR and other types of functional brain imaging, basic assumptions related with the approach should be tested. The first assumption to assess is that frontal LWIR emissions vary as a function of the cognitive load. The goal of this pilot study was to determine whether frontal LWIR emissions would significantly change during the execution of neuropsychological tests known to differentially activate the prefrontal cortex.

\section{MATERIALS AND METHODOLOGY}

Forty-seven volunteers participated in this study $(36 \%-11$ $\widehat{\delta}$, mean age: $27.4 \pm 11.0$, range 21 to 63 ; mean number of years of education: $16.8 \pm 2.4$, range 14 to 27 ). Each participant was individually assessed in a closed, mildly lit, sound-attenuated experimental room at constant ambient temperature $\left(20^{\circ} \mathrm{C}\right)$. Participants were seated behind a desk, in front of a 52 " flat screen monitor $(2 \mathrm{~m})$, connected to two control computers placed in the back of the room. Two LWIR sensitive sensors embedded in a headgear (TT-pIR HEG device, Thought Technology Ltd, Montreal) were placed at a one-inch distance from the skin on each side of the forehead midline (Fp1 and Fp2 positions of the 10-20 international EEG system corresponding to left and right prefrontal regions). The signal was transformed with a ProComp Infinity encoder, processed in real time with the Biograph software, and analysed offline with the Physiology suite (Thought Technology, Ltd). A block design experiment with counterbalanced presentations of three classic neuropsychological tasks was used with Stim2 (Neuroscan Inc, Charlotte, NC). The first task was a simple presentation of colored rectangles $(25 \times 40 \mathrm{~cm})$ placed in the center of the screen (colors: blue, red or green, presented in pseudo- 


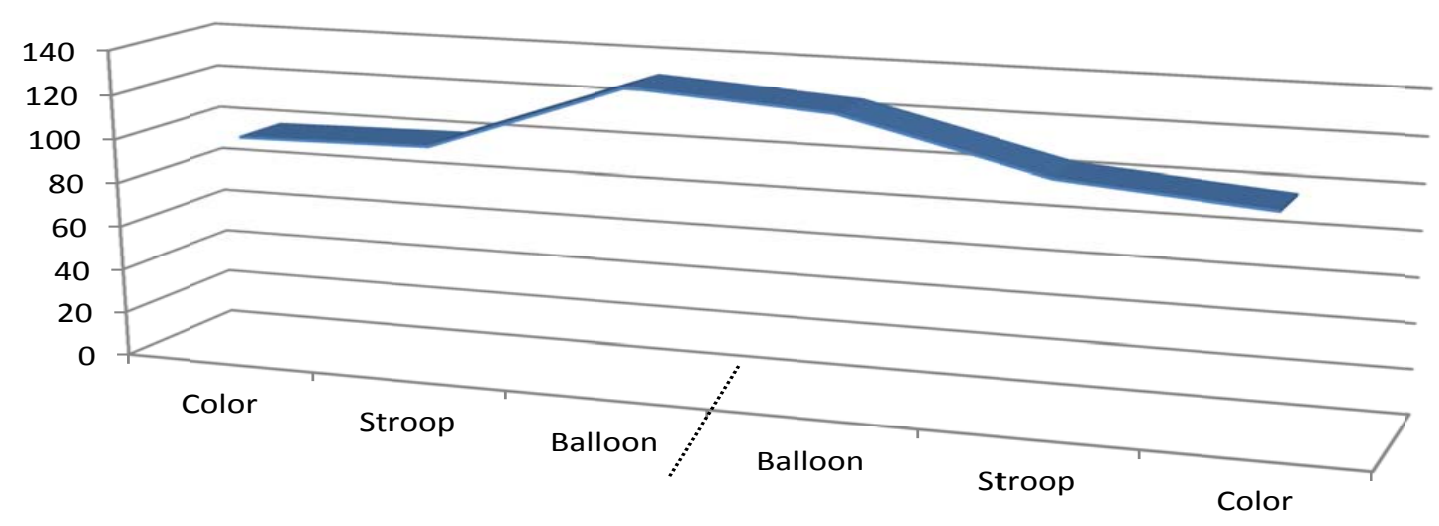

Fig. (1). Area under the curve (mean) during the three conditions after merging (dashed bar) the two subgroups of participants. (counter balanced order of presentations; $n=23$ in each order).

random order during $2000 \mathrm{~ms}$ with an inter-trial interval of $1000 \mathrm{~ms}$, 64 stimuli, total duration of approximately 5 minutes). Passive presentation of colors is classically associated with activation of posterior parts of the cortex, especially occipital regions (e.g. [14, 15]). The second task involved the well-known Stroop effect, closely associated with fronto-medial structures (most notably the anterior cingulate and the medial frontal cortices), and the posterior parietal cortex [16-18]. In the present study, a virtual version of the Stroop task was used [19], in which color blocks (blue, red or green) or color words (blue, red or green) were visually presented while names of colors (blue, red or green) were simultaneously and verbally enunciated (72 stimuli, 36 congruent and 36 non congruent; total duration of 10 minutes). Participants had to click the left (or right) button of a mouse only when visual and audio stimuli matched. The third task was an improved version of the Balloon Analog Risk Task (www.millisecond.com), a gambling game known to recruit widespread prefrontal regions, including the ventral, medial, and dorsolateral cortices [20, 21]. A balloon (blue, red or green) was presented in the center of the screen and participants had to click the left (or right) button of the mouse to put virtual air into it. Each click corresponded to a pump, which gradually inflated the balloon. The goal of the game is to maximally inflate the balloon without exploding it. The maximal number of pumps allowed before explosion varied pseudo-randomly between each trial. Each pump was associated with real money ( 25 cents) to enhance interest or anxiety related with the task, and an explosion was related with the loss of money gain for that trial (participants made $\$ 13.00$ on average, $\$ 1.25 \mathrm{~min}$ and $\$ 25.50 \mathrm{max}$, total duration of approximately $10 \mathrm{~min}$ ). Thus, the three task were chosen as a function of their various association with frontal cortex activation (lowest to highest: color bock $<$ Stroop $<$ Balloon). The order of presentation of the tasks was counterbalanced across participants so that half of the group received the color block condition first and the other half received the Balloon condition first. Given the exploratory nature of this preliminary experiment, there was no a priori hypothesis. However, if infrared sensors are sensitive to any frontal lobe activation, the average amplitude of frontal LWIR emissions (area under the curve) should significantly differ across conditions as a function of their known frontal lobe activation properties.

\section{RESULTS}

The dependant variable was the area under the curve recorded during the experiment. On average, it differed between each condition in the expected directions: color presentation $(98.2 \pm 3.1)<$ Stroop task $(102.6 \pm 26.4)<$ Balloon task $(129.2 \pm 99.3)$. The differences were significant $\mathrm{F}(2,138)=3.67, \mathrm{p}<0.05$ with a medium effect size $\left(\boldsymbol{\eta}^{2}=\right.$ 0.052 ; [22]). LSD post-hoc analyses confirmed the presence of significant differences between the Balloon and both the Color $(p=0.01)$ and the Stroop conditions $(p=0.03)$. Order of presentation had no effect upon these differences (Fig. 1). Given that variation of the variable (standard deviations) also differed across the conditions (increasing as a function of the complexity: Color $<$ Stroop $<$ Balloon), behavioral results were taken into consideration, and a significant positive correlation was found between reaction time at the Stroop task and the $\mathrm{T}$ area under the curve (2-tailed Pearson $\mathrm{r}=$ $0.46 ; \mathrm{p}<0.01)$.

\section{CONCLUSION}

These preliminary data suggest that LWIR emitted from the forehead might vary as a function of cortical frontal activation (perhaps reflecting blood flow modification), as it was first proposed by Lombard [7]. Concomitant validity studies using other brain imaging approaches with LWIR are warranted. If LWIR emitted from the scalp are truly associated with cortical activation, the use of sensors sensitive to LWIR might not only serve as a neuromodulating technique (e.g. to treat migraine; [12]), but also as a crude, yet simple, low-cost, easy-to-use instrument to evaluate cortical response in clinical settings. Only studies with larger groups of clinical and nonclinical participants will allow to test this possibility.

\section{CONFLICT OF INTEREST}

The authors confirm that this article content has no conflicts of interest. 


\section{ACKNOWLEDGEMENTS}

Declared none.

\section{REFERENCES}

[1] Carosena M. Infrared thermography: recent advances and future trends. Bentham eBooks 2012, DOI: 10.2174/97816080514341120101

[2] Arora N, Martins D, Ruggerio D, et al. Effectiveness of a noninvasive digital infrared thermal imaging system in the detection of breast cancer. Am J Surgery 2008; 196: 523-6.

[3] Kateba B, Yamamotod V, Yua C, Grundfest W, Gruena JP. Infrared thermal imaging: a review of the literature and case report. Neuroimage 2009; 47: T154-62.

[4] Villringer A, Dirnagl U. Coupling of brain activity and cerebral blood flow: basis of functional neuroimaging. Cerebrovasc Brain Metab Rev 1995; 7: 240-76.

[5] Kiyatkin EA, Brown PL, Wise RA. Brain temperature fluctuation: a reflection of neural activation. Eur J Neuroscience 2002; 16: 1648 .

[6] Schevelev IA. Functional imaging of the brain by infrared radiation (thermoencephaloscopy). Prog Neurobiol 1998; 56: 269-305.

[7] Lombard JS. Experimental researches on the regional temperature of the head under conditions of rest, intellectual activity, and emotion. London: H.K. Lewis 1879.

[8] Zago S, Ferrucci R, Marceglia S, Priori A. The Mosso method for recording brain pulsation: the forerunner of functional neuroimaging. Neuroimage 2009; 48: 652-6.

[9] Coben R, Padolsky I. Infrared imaging and neurofeedback: Initial reliability and validity. J Neurother 2007; 11: 3-13.

[10] Coben R, Myers TE. Sensitivity and specificity of Long Wave Infrared Imaging for attention-deficit/hyperactivity disorder. J Attention Disord 2009; 13: 56-65.

[11] Iznak AF, Nikishova MB. Thermoencephaloscopy of brain responses to emotionally significant visual stimuli in depressive patients. Hum Physiol 2007; 33: 370-2.

[12] Toomim H, Carmen J. Hemoencephalography: Photon-based blood flow neurofeedback. In: Budzynski TH, Budzynski HK, Evans JR,
Abarbanel A, Eds. Introduction to quantitative EEG and neurofeedback: advanced theory and applications, 2nd ed. NY: Academic Press 2009.

[13] Suktanskii AL, Yablonskiy DA. Theoretical model of temperature regulation in the brain changes in functional activity. Proc Nat Acad Sci 2006; 103: 12144-9.

[14] Beauchamp MS, Haxby JV, Jennings JE, DeYoe EA. An fMRI version of the Farnsworth-Munsell 100-Hue test reveals multiple color-selective areas in human ventral occipitotemporal cortex. Cereb Cortex 1999; 9: 257-63.

[15] Kastner S, De Weerd P, Desimone R, Ungerleider LG. Mechanisms of directed attention in the human extrastriate cortex as revealed by functional MRI. Science 1998; 282(5386): 108-11.

[16] Bush G, Whalen PJ, Rosen BR, Jenike MA, McInerney SC, Rauch SL. The counting stroop: an interference task specialized for neuroimaging-validation studies with functional MRI. Hum Brain Mapp 1998; 6: 270-82.

[17] Peterson BS, Skudlarski P, Gatenby JC, Zhang H, Anderson AW, Gore JC. An fMRI study of stroop word-color interference: evidence for cingulate subregions subserving multiple distributed attentional systems. Biol Psychiatr 1999; 45: 1237-58.

[18] Pujol J, Vendrell P, Deus J, et al. The effects of medial frontal and posterior parietal demyelinating lesions on Stroop interference. Neuroimage 2001; 13: 68-75.

[19] Henry M, Joyal CC, Nolin P. Development and initial assessment of a new paradigm for assessing cognitive and motor inhibition: the bimodal virtual-reality Stroop. J Neurosci Methods 2012; 210: 12531.

[20] Fecteau S, Pascual-Leone A, Zald DH, et al. Activation of prefrontal cortex by transcranial direct current reduces appetite for risk during ambiguous decision making. J Neurosci 2007; 27: 6212-8.

[21] Rao H, Korcykowski M, Pluta J, Hoang A, Detre JA. Neural correlates of voluntary and involuntary risk taking in the human brain: an fMRI study of the Balloon Analog Task (BART). Neuroimage 2008; 42: 902-10.

[22] Cohen J. Statistical power analysis for the behavior sciences, 2nd ed. Hillsdales NJ: Lawrence Elbaum Associated 1988.

(C) Joyal and Henry; Licensee Bentham Open.

This is an open access article licensed under the terms of the Creative Commons Attribution Non-Commercial License (http://creativecommons.org/licenses/by$\mathrm{nc} / 3.0 /$ ), which permits unrestricted, non-commercial use, distribution and reproduction in any medium, provided the work is properly cited. 